\title{
L'efficacité de la formation continue des enseignants du primaire : le cas du Burkina Faso ${ }^{1}$
}

Cégep de Rivière-du-Loup

Clemont Gauthier

Université Laval

\author{
The effectiveness of continuing professional \\ development for primary school teachers: \\ The case of Burkina Faso
}

doi:10318162/fp.2017.330

\section{ésumé}

Pour honorer ses engagements internationaux, le Burkina Faso a eu recours, à l'instar de nombreux autres pays en développement, à des recrutements massifs d'enseignants qui, selon plusieurs analystes, ont compromis la qualité de léducation pour tous. Face au plafonnement des rendements scolaires et aux besoins de nouvelles compétences pédagogiques créés par différents contextes difficiles de scolarisation, la nécessité d'améliorer le système de formation continue fait consensus. Le présent article prend appui sur une recherche inspirée du modèle de formation continue efficace de Guskey (2000) et dont l'objectif était de proposer le profil des activités de formation pouvant soutenir une éducation de qualité pour tous au Burkina Faso. Grâce à une analyse comparative des descriptions ethnographiques de huit circonscriptions scolaires (quatre à très fort taux de réussite et quatre autres à très faible taux), nous dégageons le profil de la formation continue orientée sur la réussite scolaire des élèves. Une discussion permet de valider les résultats obtenus.

Mots-clés

Formation continue, réussite scolaire, Burkina Faso, efficacité

Abstract

To honor its international commitments, Burkina Faso, like many developing countries, has resorted to massive recruitments of teachers. According to various analysts, such recruitments have compromised the quality of education for all. There is a consensus on the need to improve the system of continuing professional development for teachers, in particular when faced with a levelling off of student achievement and with the need for new pedagogical skills in challenging educational contexts. This article is based on a research inspired by Guskey's (2000) model of effective professional development, with the objective of proposing the profile of professional development activities likely to support quality education for all in Burkina Faso. The comparative analysis of ethnographic descriptions of eight school districts (four with high achievement rates and four with very low rates) allows us to draw the profile of student achievement-focused professional development. We close the article with a discussion aiming to validate the results of this analysis.

\section{Keywords}

Teacher development, school achievement, Burkina Faso, school effectiveness

\section{Introduction}

\section{Le besoin de la requalification des enseignants du primaire au Burkina Faso}

Après la conférence de Jomtien en 1990 et le Forum mondial sur l'éducation en 2000, l'euphorie de «l'éducation pour tous en l'an 2015 » a poussé plusieurs pays africains, avec la bénédiction d'instances internationales, à recruter massivement des enseignants et à construire des écoles pour élever le taux de scolarisation au primaire. Tel a été également le cas au Burkina Faso. La poursuite de l'Éducation pour tous (EPT) s'est donc traduite par un développement des infrastructures scolaires et une forte demande de nouveaux enseignants. D'ailleurs, selon le dernier rapport de l'EPT (UNESCO, 2014, p. 224), le déficit quantitatif d'enseignants au Burkina Faso ne pourrait être résorbé avant 2030. Les collaborateurs du Rapport mondial de suivi sur l'EPT 2005 en sont venus à se demander si l'aspect quantitatif de l'EPT ne prenait pas le pas sur sa qualité, car «se borner à remplir d'enfants des espaces appelés "écoles" ne répondrait même pas aux objectifs quantitatifs si aucune éducation réelle n'y était dispensée "(UNESCO, 2005, p. 31). L'EPT vise à « améliorer la qualité de l'éducation sous tous ses aspects de façon à obtenir des résultats d'apprentissage notamment en ce qui concerne la lecture, l'écriture, le calcul et les compétences indispensables dans la vie courante »(UNESCO, 2005, p. 31).

Depuis, les rapports de l'EPT de l'UNESCO se succèdent et identifient, parmi les multiples défis à l'obtention des résultats satisfaisants au primaire, le besoin de formation continue des enseignants qui apparait au deuxième des quatre axes du dernier rapport : recruter de meilleurs candidats, bien former les enseignants avant et pendant leur carrière, offrir des incitatifs pour attirer des enseignants en zones défavorisées et améliorer les conditions de travail (UNESCO, 2014, p. 187). 
Le Burkina Faso n'échappe pas à ce besoin. Comme d'autres pays d'Afrique, le déficit d'enseignants avait créé, pendant plus d'une décennie, la légitimité sociale d'envoyer dans les écoles du personnel ayant peu de formation pédagogique. En effet, au terme de la formation initiale reçue dans les écoles nationales des enseignants du primaire (ENEP), la formation pédagogique est à peine suffisante. D'ailleurs, Kyelem et Barro (2007) et Beogo (2014) constatent, malgré quelques avancées, la pénurie de formation de formateurs d'enseignants et le peu de ressources pour un meilleur suivi des enseignants stagiaires $^{2}$.

À la sortie de l'ENEP, l'enseignant burkinabè fait face à une réalité scolaire complexe où cohabitent la malnutrition, l'absence de motivation et la non-maîtrise de la langue d'enseignement, réalités qui pèsent sur l'apprentissage des élèves (Kamano, Rakotomalala, Bernard, Husson et Reuge, 2010). Les enseignants nont pas toujours le loisir d'enseigner dans des classes dites standards quant à leur taille, à leur composition et à leur localisation. En effet, le Burkina Faso compte plusieurs écoles présentant des caractéristiques diverses : effectifs pléthoriques, classes multigrades, écoles bilingues ou éloignées des grands centres. Dans un tel contexte, les compétences pédagogiques des nouveaux enseignants sont donc mises à rude épreuve, notamment dans les zones rurales. En effet, les nouveaux enseignants évitent les zones rurales (Kyelem et Barro, 2007), souvent reculées, qui riment avec un soutien relatif des parents envers la scolarisation de leurs enfants (achat des fournitures scolaires, suivi et encouragements liés aux travaux scolaires).

Dans l'objectif d'une éducation de qualité pour tous qui permettrait au Burkina Faso de sortir de « la crise de l'apprentissage »(UNESCO, 2014, p. 186), comment outiller les enseignants du primaire audelà du bagage pédagogique acquis au terme de la formation initiale?

\section{Pratiques de la formation continue des enseignants au Burkina Faso}

Au Burkina Faso, la réponse est donnée par une foule d'activités de formation : groupes d'animation pédagogique (GAP), quinzaines critiques, conférences pédagogiques et stages de recyclage sont mis en branle chaque mois ou chaque année. Selon les évaluations qu'en font Diallo (1990), Ilboudo et al. (2001), Batiana (2002) et dernièrement Beogo (2014), ces activités de formation sont dominées par des présentations d'informations, des conférences politisées, des discussions stériles sans apprentissages pratiques. Ces constats nous poussent à exprimer plusieurs interrogations générales : à quels objectifs correspondent ces activités de formation? Quelles pratiques pédagogiques privilégie-t-on? Quels résultats d'apprentissage attend-on de ces activités? En résumé, quels sont les facteurs d'efficacité des activités de formation continue au Burkina Faso?

\section{Cadre conceptuel}

Comme le souligne Guskey: «the most crucial evidence on the effectiveness of professional development [is] improvements in students learning » (2000, p. 12). C'est dans cette perspective que la réponse aux questions de recherche sera analysée.

Par ailleurs, Boatman (2003), Craig, Kraft et Du Plessis (1998), Guskey (2000, 2001), Guskey et Huberman (1995), Joyce et Showers (1988), Killion (2002), Sousa et Donovan (1993) ainsi que 
Sparks et Loucks-Horsley (1989) soutiennent et démontrent également que les activités de formation pourraient s'organiser selon la finalité liée à la réussite scolaire. Leurs analyses ont permis à Guskey de modéliser ainsi l'effet de la formation continue sur les résultats académiques des élèves (figure 1) :

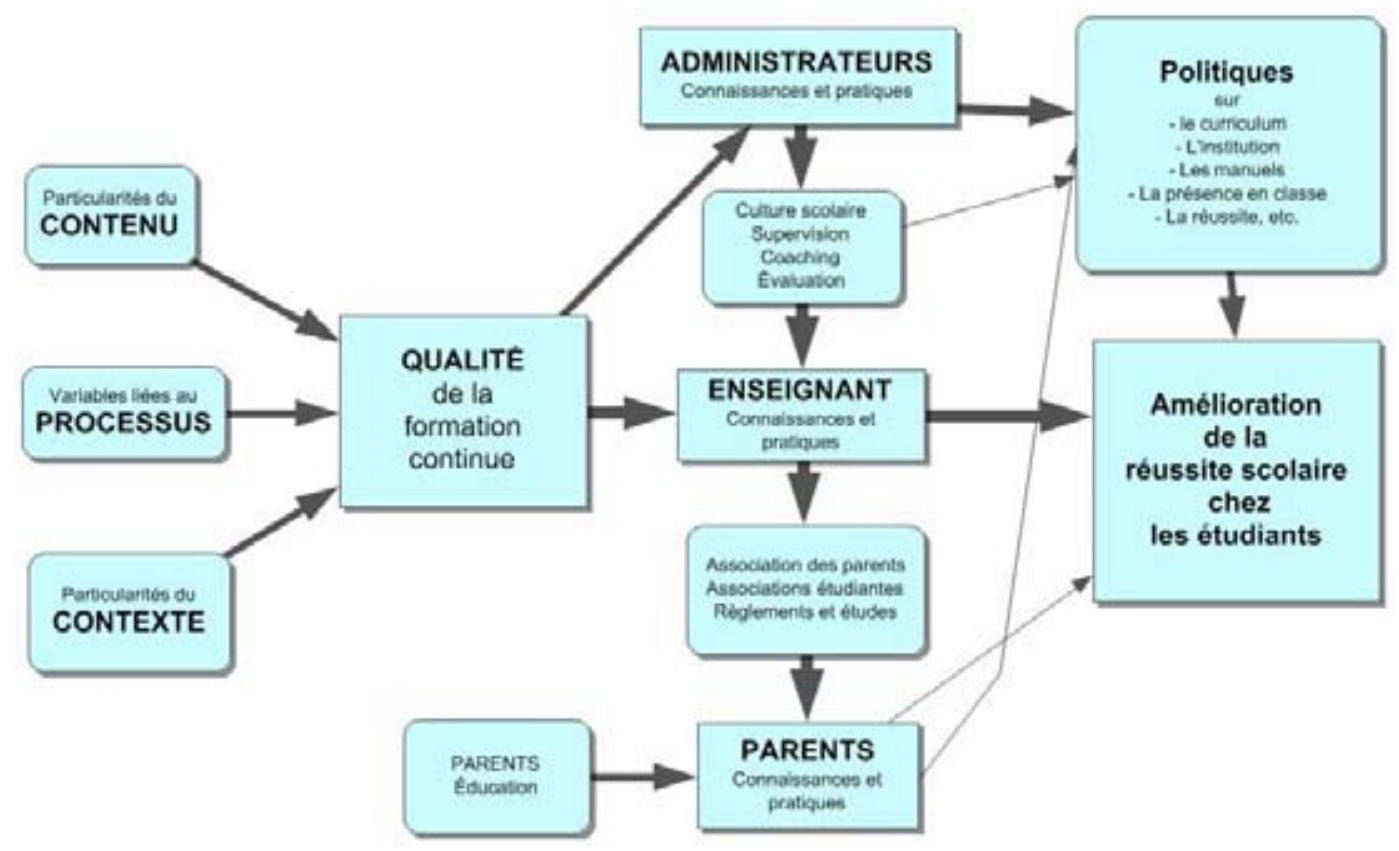

Source : Guskey (2000), p. 73.

\section{Figure 1}

Modélisation du lien entre la formation continue et la réussite scolaire.

Selon la modélisation ci-dessus, la formation continue ne produit pas d'effets directs d'amélioration chez l'élève. Elle est d'abord reliée à la variable « enseignant " par les connaissances et les pratiques de celui-ci. Ces connaissances et pratiques constituent ainsi les particularités du contenu de la formation. En plus du contenu, la formation devrait s'articuler selon un processus et un contexte. Par ailleurs, la variable «enseignant» n'agit pas seule. L'enseignant est soutenu par une structure administrative (administrateurs) qui lui fournit un cadre pédagogique (politique institutionnelle sur l'évaluation des élèves, la présence des élèves en classe, la langue...) et un cadre d'amélioration de sa pratique professionnelle (supervision, coaching...). Enfin, les administrateurs et l'enseignant sont aidés par une communauté locale (parents) dont les influences ne sont pas à négliger dans un contexte rural.

S’inspirant de ce modèle, Guskey $(2000,2001)$ dégage cinq composantes essentielles permettant à toute activité de formation continue d'influencer positivement la réussite scolaire :

1. politiques d'enseignement (valorisées lors des activités de formation);

2. pratiques d'enseignement (enseignées dans les activités de formation);

3. stratégies d'organisation des activités (le lieu, la durée de la formation); 
4. connaissances et des habiletés (à acquérir par l'enseignant à la fin des activités de formation);

5. soutiens organisationnels (prises en charge par l'administration et la communauté locale).

Notons que ce modèle est régulièrement utilisé pour apprécier l'efficacité des activités de formation continue : en 2005, par Brodeur et al.; en 2010, par Bennison et Goos; en 2013, par Claesgens et al. ainsi que par Fontaine, Savoie-Zajc et Cadieux; en 2014, par Lewis, Baker, Watts et Lang ainsi que par Williams-McMillan et Hauser; en 2017, par Zambak, Alston, Marshall et Tyminski.

Le modèle de Guskey s'appuie sur cinq composantes. Cependant, au Burkina Faso, nous ne pouvons parler de conditions d'efficacité des activités de formation sans considérer une sixième composante, la participation des enseignants à celles-ci. En effet, Diallo (1990), Ilboudo et al. (2001), Maïga (1995) et, tout récemment, Beogo (2014, p. 190) soulignent encore le manque de motivation et l'absentéisme des enseignants aux activités de formation. Nous bonifions donc ce modèle d'une sixième composante, la participation des enseignants.

À la lumière de ce cadre théorique, nous nous demandons plus précisément comment les activités de formation continue au Burkina Faso peuvent être efficaces selon les politiques qu'elles valorisent, les pratiques d'enseignement qu'elles préconisent, l'organisation et les soutiens dont elles bénéficient, les connaissances et habiletés qu'elles font acquérir et les stratégies dont elles usent pour maximiser la participation des enseignants.

\section{Méthodologie de la recherche}

\section{Une approche descriptive}

Notre démarche méthodologique s'appuie donc sur une description. À la manière d'un ethnométhodologue (Le Breton, 2004), notre intention est d'interroger plusieurs acteurs capables de décrire les pratiques liées aux activités de formation afin que nous puissions y tirer un portrait systémique de la formation continue efficace. Pour dégager ce portrait systémique, nous faisons appel à ce qu'Huberman et Miles (1991) appellent « l'accumulation de cas comparables », une accumulation qui aboutit à un profil commun et latent par saturation des données.

\section{Une approche par comparaison intersites}

En quoi le profil commun dégagé témoignera-t-il de l'«efficacité » de la formation continue? Afin d'obtenir des données plus probantes, nous avons opté pour une comparaison entre les cas de formation en zones de forte réussite scolaire et les cas de formation en zones de faible réussite. Cela nous donnera des pistes sur ce qui semble faire une différence. Cette méthode de comparaison intersites est bien décrite par D'Amboise et Audet (1996), Karsenti et Demers (2000) ainsi que Tessier et D'Amboise (2001).

Pour opérationnaliser la réussite scolaire, nous avons modifié la formule de classification des écoles secondaires du Québec de l'Institut Fraser $^{3}$ pour l'appliquer aux taux de réussite aux examens du ministère d'au moins 60 \% des circonscriptions du Burkina Faso. Nous avons choisi 8 circonscriptions, dont 4 à très fort taux de réussite et 4 autres à faible taux. 
Dans chacune des circonscriptions, nous avons mené des entrevues non directives auprès des conseillers pédagogiques et des entrevues semi-directives auprès des directeurs d'école et des enseignants dans le but de constituer des descriptions des activités de formation continue. Voici le tableau synoptique :

\begin{tabular}{|l|l|l|}
\hline $\begin{array}{l}\text { Circonscriptions } \\
\text { à faible taux de réussite scolaire }\end{array}$ & $\begin{array}{l}\text { Circonscriptions } \\
\text { à taux élevé de réussite scolaire }\end{array}$ & Totaux \\
\hline 4 conseillers pédagogiques & 4 conseillers pédagogiques & $\mathbf{8}$ conseillers \\
\hline 8 directeurs d'école & 8 directeurs d'école & $\mathbf{1 6}$ directeurs \\
\hline 17 enseignants dont & $\begin{array}{c}17 \text { enseignants dont } \\
-\quad \text { ceux du CM1 et CM2; }\end{array}$ & $\mathbf{3 4}$ enseignants \\
$-\quad$ l'enseignant le plus ancien; & - l'enseignant le plus ancien; & \\
$-\quad$ représentation féminine. & - représentation féminine. & \\
\hline
\end{tabular}

\section{Tableau 1}

Répartition des participants.

Chaque entrevue enregistrée a duré entre quarante-cinq minutes et une heure. Enfin, les observations sur le terrain et les documents obtenus ont servi à confirmer plusieurs informations émanant des entrevues.

\section{La démarche d'analyse qualitative}

Après avoir saisi les verbatims de 58 entrevues, nous avons réalisé une catégorisation mixte (catégories prédéterminées et émergentes) des unités de sens (L'Écuyer, 1990; Merriam, 1998). Les catégories prédéterminées sont les six composantes de la formation continue. Les catégories émergentes ont été sélectionnées à partir des mots ou des expressions ayant les occurrences les plus élevées. Chaque catégorie émergente a fait l'objet d'une description par saturation (Huberman et Miles, 1991). Nous avons ainsi abouti au profil général des activités de formation continue dans les circonscriptions à taux élevé de réussite scolaire et celui des circonscriptions à faible taux de réussite scolaire. Selon la méthode de comparaison intersites (D'Amboise et Audet, 1996; Tessier et D'Amboise, 2001), nous avons mis en parallèle les deux profils et retiré les similitudes pour ne conserver que les résultats spécifiques aux circonscriptions à fort taux de réussite.

\section{Le corpus d'études servant à la discussion des résultats}

Une fois les résultats spécifiques obtenus, nous avons passé chaque résultat sous la loupe d'un corpus de 10 méta-analyses, 7 recherches de troisième niveau (Ellis, 2001) et 23 synthèses de revue de littérature scientifique de troisième niveau et 14 recherches de deuxième niveau. L'ensemble de la démarche pourrait être ainsi résumé dans le tableau suivant. 


\begin{tabular}{|c|c|c|c|}
\hline $\begin{array}{l}\text { Identification des sites } \\
\text { de reproductions } \\
\text { théorique et littérale }\end{array}$ & $\begin{array}{l}\text { Profils systémiques } \\
\text { par accumulations de } \\
\text { cas comparables }\end{array}$ & $\begin{array}{c}\text { Comparaison } \\
\text { intersites des } \\
\text { profils des deux } \\
\text { groupes }\end{array}$ & $\begin{array}{l}\text { Comparaison } \\
\text { Corpus/terrain }\end{array}$ \\
\hline $\begin{array}{l}\text { Reproduction littérale } \\
\text { sur plusieurs sites } \\
\text { (présence de réussite } \\
\text { scolaire) }\end{array}$ & $\begin{array}{c}\text { Composantes des } \\
\text { programmes de } \\
\text { formation continue des } \\
\text { cas efficaces }\end{array}$ & \multirow[b]{2}{*}{$\begin{array}{l}\text { Extraction des } \\
\text { composantes } \\
\text { potentiellement } \\
\text { efficaces }\end{array}$} & \multirow{2}{*}{$\begin{array}{c}\text { Validation } \\
\text { externe des } \\
\text { composantes de } \\
\text { formation } \\
\text { continue efficace }\end{array}$} \\
\hline $\begin{array}{l}\text { Reproduction théorique } \\
\text { sur plusieurs sites } \\
\text { (absence de réussite } \\
\text { scolaire) }\end{array}$ & $\begin{array}{c}\text { Composantes des } \\
\text { programmes de } \\
\text { formation continue des } \\
\text { cas inefficaces }\end{array}$ & & \\
\hline
\end{tabular}

Tableau 2

Démarche globale de la comparaison intersites.

\section{Résultats et discussions selon les composantes de la formation continue dite efficace}

Les six composantes de la formation continue servent de cadre de présentation des résultats. Même si nous avons comparé deux groupes de circonscriptions, nous présentons uniquement les résultats qui font la différence.

\section{Première composante : I'efficacité de la formation continue par les politiques d'enseignement}

Au Burkina Faso, les circonscriptions à forte réussite scolaire se démarquent de celles à faible réussite par la mise en place d'activités de formation orientées par les plans d'amélioration individuelle (PAI) et collective (PAC) :

J'applique le plan d'amélioration fréquemment en lecture et de temps en temps en calcul [...]. C'est le PACEB qui, à l'époque, avait proposé ça. Cela a été quand même appliqué presque de façon générale. Ça demande un travail supplémentaire. Il faut faire une évaluation dans la discipline-clé tous les jours (CEF3, enseignante, 14 ans d'expérience).

Les plans d'amélioration individuelle et collective tablent sur l'amélioration de l'apprentissage de la langue d'enseignement chez les élèves. En effet, ces circonscriptions efficaces ont constaté que «la priorité des priorités » est l'apprentissage de la lecture (Batiana, 2002; Brodeur et al., 2005; National Early Literacy Panel, 2008; National Institute of Child Health and Human Development [NICHHD], 2000). Par une priorité accordée à la réussite, le défi pédagogique lancé aux écoles à effectifs pléthoriques ou dans des situations particulières devient quotidien, sans l'attente d'un financement quelconque.

Killion (2002), Lane (2003) et Womack (2004) soulignent la nécessité de déterminer des standards de réussite. Pour leur part, Harshman et Yezierski (2017) de même que Fontaine, Savoie-Zajc et Cadieux (2013) montrent les bénéfices de la traduction des standards en évaluations formatives continues 
en classe. Ces standards de réussite, recommandés par les ministères de l'Éducation, sont assortis d'évaluations régulières et d'indicateurs d'atteinte de la maîtrise de la langue d'enseignement.

\section{Deuxième composante : l'efficacité de la formation continue par des pratiques d'enseignement}

Au Burkina Faso, dans les zones à fort taux de réussite scolaire, comparativement à celles à faible taux, les activités de formation continue diffèrent par la pratique répétée de la pédagogie de groupe dans les classes à effectifs pléthoriques, la pratique des évaluations diagnostiques et formatives régulières en lecture et l'enseignement direct du vocabulaire :

On se retrouve avec une centaine d'élèves alors que les classes ne répondent pas au nombre conçu depuis le décret de 1989 qui est jusqu'à 70-80 élèves. Alors si tu te retrouves dans une classe avec 100 élèves [...]. Disons que dans toutes les disciplines, les gens travaillent en groupe $[\ldots]$. Nous leur donnons un temps pour se retrouver en groupe [...]. Le travail de groupe n'est pas facile puisqu'il faut forcément dans chaque groupe deux ou trois élèves qui se débrouillent mieux. C'est généralement en lecture que je fais le travail de groupe, également les leçons d'éveil histoire, science, géographie. Le chef de groupe apprend et récite sa leçon devant les autres membres du groupe. Il interroge chaque membre. Lélève signale ceux qui ne savent pas leur leçon (CEF, enseignante, 4 ans d'expérience).

Bowman-Perrott et al. (2013), Hattie (2003), Kunsch, Jitendra et Sood (2007) ainsi que Lou, Abrami et D'Apollonia (2001) et Lou et al. (1996) insistent sur la formation des enseignants et des élèves, le bon dosage de la composition et de la taille des groupes, la fréquence et le respect des étapes de la pédagogie de groupe.

Une autre pratique d'enseignement jugée efficace au Burkina Faso dont Graves (1986), le National Early Literacy Panel (2008) et le NICHHD (2000) se font l'écho est le diagnostic systématique des élèves possiblement en difficulté en début et en cours d'année. Au Burkina Faso, dans l'apprentissage $\mathrm{du}$ français, l'enseignant pratique le diagnostic par les pairs par le biais de la lecture en groupes. Cette pratique consiste à demander à l'élève expert et responsable du groupe de signaler les camarades éprouvant des difficultés.

Dans le cas d'une cohorte homogène et faible, les enseignants imposent l'application de la méthode syllabique par des jeux d'éveil de la conscience phonologique, par un renforcement de la conscience graphophonologique et par l'enseignement direct du vocabulaire, ce qui produit de meilleurs résultats (Edgerton, 2000; Graves, 1986; Gray, Drummond et Greenhill, 2009; Lewis et al., 2014; National Early Literacy Panel, 2008; NICHHD, 2000).

Dans les circonscriptions à fort taux de réussite scolaire, ce qui fait la différence en mathématiques porte sur la formation de l'enseignant à la pratique régulière d'une même séquence didactique : les phases de révision, de motivation et de concrétisation. La plupart des enseignants disent la pratiquer régulièrement. Quant au matériel de concrétisation, ce n'est pas uniquement l'utilisation d'outils extérieurs; cela comprend l'illustration de certains concepts mathématiques (Baker, Gersten et Lee, 2002) et la pratique de résolutions de problèmes (Adey, 1995; Lane, 2003; Rudnitsky, Etheredge, Freeman et Gilbert, 1995). En effet, la présentation d'un large éventail d'exemples de concepts et de problèmes permet aux élèves de conceptualiser par regroupement et par différenciation. 
En sciences, certaines circonscriptions insistent sur l'observation libre. Cependant Lou et al. (2001) la déconseillent, car son utilisation cause des pertes de temps et de la dissipation chez les élèves, tandis que l'observation dirigée permet une plus nette avancée : c'est le principe de l'enseignement direct ou explicite (Barlow, Frick, Barker et Phelps, 2014; Claesgens et al., 2013; Dewi, Kultsum et Armadi, 2017; Hattie, 2003; Richard, Bissonnette, Castonguay et Gauthier, 2013). L'enseignant qui maîtrise cette technique en arrive à une prise en charge rapide des plus faibles.

\section{Troisième composante : I'efficacité de la formation continue par les connaissances et les habiletés de l'enseignant}

Au Burkina Faso, la différence marquée par les zones à taux élevé de réussite porte sur des activités de formation continue qui développent les connaissances disciplinaires de la matière à enseigner et les connaissances psychologiques concernant les élèves. Ces activités renforcent d'autres habiletés de base comme la formulation des objectifs pédagogiques et les items d'évaluation. Dans notre corpus documentaire, les études confirment que la connaissance disciplinaire est souvent mise à mal quand sévissent de mauvaises politiques de recrutement d'enseignants. Quand le niveau de scolarité à l'entrée des écoles de formation est très bas, Lane (2003) et Rolheiser-Bennett (1986) accordent une priorité à la mise à niveau des enseignants dans la discipline concernée. L'expertise des enseignants en arrive alors à pallier l'absence des manuels scolaires. Cette expertise sort l'enseignant de l'obéissance servile aux manuels scolaires pour une meilleure adaptation des textes de lecture aux réalités locales (Boatman, 2003; Killion, 2002). Dans les études comme dans l'échantillon, les plus aguerris rédigent leurs propres textes destinés à la lecture en y insérant des mots désignant des objets de la réalité socioculturelle immédiate de l'école :

Donc je me suis dit, «pourquoi ne pas élaborer des textes supports pour le vocabulaire au CE1, CE2? ». Les mots sont tellement dispersés (dans le manuel) que certains enseignants les prennent et les plaquent tout simplement au tableau. Normalement un texte, on doit pouvoir l'exploiter. Quand on voit le contexte dans lequel le mot se trouve, on peut saisir à peu près la signification. Donc j'ai sacrifié des vacances pour faire quarante-huit textes liés aux quarante-huit thèmes (CEM2, directeur, 22 ans d'expérience).

L'habileté maître de l'enseignant efficace demeure la pratique continuelle de l'évaluation formative (Harshman et Yezierski, 2017) tandis que l'attitude est l'esprit de collaboration (Adey, 1995; Lewis et al., 2014).

\section{Quatrième composante : l'efficacité de la formation continue par les stratégies d'organisation des activités}

La durée optimale de formation, le dosage entre contenu théorique et pratique, l'expertise des encadreurs, le suivi, lévaluation et la fidélité des implantations sont les atouts majeurs des activités de formation. Les activités « one-shot » seraient à abandonner (Craig et al., 1998; Guskey et Huberman, 1995; OCDE, 1998; Solar, 2001; Sparks et Loucks-Horsley, 1989). En effet, les changements de pratique chez les enseignants prennent du temps. Quels que soient les compétences à développer ou les objectifs d'amélioration dans la formation, la littérature distingue une phase d'acquisition conceptuelle, une phase de pratiques guidées et une phase de personnalisation ou d'institutionnalisation par des suivis et des évaluations portant sur les effets attendus (Adey, 1995; Boatman, 2003; Edgerton, 2000; 
Killion, 2002; Lane, 2003). Selon Hill, Bicer et Capraro (2017), la supervision ou le tutorat sont les outils de cette personnalisation.

Le nombre élevé d'encadreurs a été associé à une plus grande efficacité de la formation continue. Sur le terrain, cette supériorité est due à la présence d'enseignants et de directeurs déchargés. Dans la littérature, plusieurs formations sont menées par des enseignants qui n'ont pas forcément le titre de capacité des conseillers pédagogiques ou d'inspecteurs, mais qui sont expérimentés ou experts dans une pédagogie particulière (Boatman, 2003; Killion, 2002). Cette abondance d'encadreurs permet davantage de pratiques regroupées par discipline ou par proximité géographique (Killion, 2002; Womack, 2004) et plus de suivis (Boatman, 2003; Edgerton, 2000; Hill et al., 2017; Killion, 2002).

\section{Cinquième composante : les stratégies participatives aux activités de formation}

Les enseignants sont toujours moins nombreux quand ils doivent participer à des activités en dehors de l'école. Dans les circonscriptions qui font la différence, la proximité des lieux de formation permet de réduire leurs dépenses financières. De plus, la plupart des activités de formation aboutissant à des changements de pratique se déroulent sur le site et permettent, de facto, la participation des enseignants de l'école choisie :

Nous avons une autre activité qu'on appelle la quinzaine critique. Tous les quinze jours, on essaye de se retrouver pour que chacun dise les difficultés qu'il vit dans sa classe et, s'il y a lieu, tous les maîtres se déportent un jour dans ladite classe pour suivre le maître. Après, on prodigue les conseils nécessaires [...]. C'est pratiquement une demi-journée (CEM54, directeur, 14 ans d'expérience).

Cette proximité peut être satisfaite quand les encadreurs ont plus de disponibilité. Quand la formation est éloignée, la prise en charge d'enseignants sélectionnés aboutit à une formation en cascade ${ }^{4}$. L'étude de Glover et al. (2016) souligne la préférence des enseignants pour la formation à même la salle de classe, quel que soit le milieu d'appartenance de l'enseignant.

À l'exemple de ce qui a été noté dans la première composante, les enseignants participent davantage aux activités quand la circonscription se donne des objectifs ou des standards de réussite (les plans d'amélioration collectifs et individuels). Dans les classes terminales du primaire, la priorité des plans d'amélioration reste la réussite aux examens ministériels. Certaines recherches soulignent que les enseignants motivés sont souvent prêts à se donner eux-mêmes des défis d'amélioration en participant à des projets institutionnels ou universitaires (Barlow et al., 2014; Kulinna, McCaughtry, Martin et Cothran, 2011; Rodriguez, 2010).

\section{Sixième composante : l'efficacité de la formation continue par des soutiens organisationnels}

Les circonscriptions à taux de réussite élevé font la différence par des soutiens clés tels l'amélioration du ratio manuel/élève et l'accessibilité aux manuels didactiques. L'appui des parents permet d'acheter des fournitures scolaires ou de payer le déplacement lors d'une formation extrasite. Les associations des mères éducatrices (AME) montrent, ici, un autre type de soutien : 
L'AME est une structure de soutien, au côté de celle des parents d'élèves, qui œuvre [pour] le suivi des filles, leur maintien à l'école, un appui à porter à celles qui sont en difficulté et la sensibilisation des parents de ces dernières pour qu'en famille, la petite fille soit déchargée des occupations domestiques qui les empêche de réussir à l'école (CEM53, conseiller pédagogique, première année).

Le soutien des parents motive les enfants, stimule leur apprentissage, ce qui concourt à améliorer le taux de réussite.

Du côté des enseignantes, l'absence du foyer pour assister à des activités de formation est plus difficile. Cependant, les circonscriptions à fort taux de réussite scolaire se démarquent par le soutien de l'entourage immédiat de l'enseignante (maris, collègues et élèves). Dans notre corpus d'études majoritairement réalisées dans les pays développés, cette problématique n'a pas été soulevée, mais la formation à distance peut aider si l'accès à la technologie est assuré et encadré à l'intérieur d'une "communauté d'apprentissage professionnelle» (Claesgens et al., 2013; Fontaine, Savoie-Zajc et Cadieux, 2013; Lewis et al., 2014).

\section{Conclusion}

Les acquis de la formation continue efficace

Une formation initiale n'est jamais suffisante et la formation continue restera toujours une nécessité. Une question demeure quant à son efficacité à contribuer à une éducation de qualité. À la lumière des résultats présentés, quel est le profil de la formation continue efficace au Burkina Faso?

Sous l'angle de la première composante, dans les meilleures circonscriptions, la formation continue est efficace si elle est portée par des objectifs de réussite dans la langue d'enseignement et aux examens du ministère.

Pour atteindre ces objectifs dans la deuxième composante, les circonscriptions à fort taux de réussite scolaire maximisent la rétroaction directe à l'élève par toutes formes de pratiques d'enseignement : pédagogie de sous-groupes, observation directe, révision et concrétisation.

Sans surprise, dans la troisième composante, le contenu de formation qui ressort du lot de ce que l'enseignant doit maîtriser pour offrir une bonne rétroaction à l'élève est la maîtrise excellente de la langue d'enseignement, des disciplines et de l'évaluation objective et formative.

Les résultats sur la quatrième composante montrent que l'enseignant, dans les circonscriptions qui se distinguent, se situe, comme l'élève, dans une formation ou un apprentissage de proximité : nombre élevé d'encadreurs et rétroaction en collégialité aboutissant à un réinvestissement immédiat.

La cinquième composante, la motivation des enseignants à participer à des activités de formation continue, est améliorée par la poursuite d'objectifs communs d'amélioration et par la formation intrasite.

Enfin, le soutien financier et psychologique de la communauté locale et de l'entourage immédiat des enseignants, et surtout des enseignantes, influencent les variables d'efficacité de la formation continue : participation, motivation, accès à des ressources didactiques et matérielles, persévérance.

44 - Formation et profession 25(2), 2017 


\section{Les limites de cette recherche}

Cette recherche a été réalisée en 2010. Elle se base sur le modèle d'efficacité de la formation continue de Guskey (2000) bonifié par l'ajout d'une sixième composante. Il reste tout à fait pertinent de collecter de nouvelles données selon d'autres modèles explicatifs, par exemple celui d'Houpert (2005).

\section{Notes}

1 Les travaux de recherche ont été réalisés grâce à une subvention du Centre de recherches pour le développement international, Ottawa, Canada.

2 Vous trouverez une description plus détaillée de la formation initiale au Burkina Faso dans Djibo, F. (2010). L’impact de la formation continue des enseignants sur la réussite scolaire: Regard critique sur le cas du Burkina Faso (Thèse de doctorat, Université Laval).

$3 \mathrm{RC}[\mathrm{Z}=(30 \mathrm{Z1a}+30 \mathrm{Z} 1 \mathrm{~b}-15 \mathrm{Z} 2 \mathrm{a}-15 \mathrm{Z} 2 \mathrm{~b}+5 \mathrm{Z3} \mathrm{a}+5 \mathrm{Z3b}) / 100)]$. (Z1a et $\mathrm{Z1b})$ sont les scores standardisés des deux taux bruts de réussite et de promotion de chaque circonscription (la réussite et promotion) sur six ans. (Z2a et Z2b) sont les scores standardisés des pentes de régression linéaire des deux taux bruts de réussite et de promotion sur six ans (le progrès) sur six ans. (Z3a et Z3b) sont les scores standardisés des écarts moyens absolus entre le taux des garçons et celui des filles dans la réussite et la promotion (la parité) sur six ans.

4 La formation en cascade consiste à former un groupe de personnes qui vont en former d'autres. C'est le principe qui sous-tend la formation des formateurs.

\section{Références}

Adey, P. S. (1995). The effects of a staff development program: The relationship between the level of use of innovative science curriculum activities and student achievement. Repéré à http://files.eric.ed.gov/fulltext/ED383567.pdf

Baker, S., Gersten, R. et Lee, D.-S. (2002). A synthesis of empirical research on teaching mathematics to low-achieving students. The Elementary School Journal, 103(1), 51-73. http://dx.doi.org/10.1086/499715

Barlow, A. T., Frick, T. M., Barker, H. L. et Phelps, A. J. (2014). Modeling instruction: The impact of professional development on instructional practices. Science Educator, 23(1), 14-26. Repéré à http://files.eric.ed.gov/fulltext/EJ1034755.pdf

Batiana, A. (2002, novembre). Études comparatives des écoles satellites 1999-2002. Communication présentée au colloque «La recherche face aux défis de l'éducation au Burkina Faso ", Ouagadougou.

Bennison, A. et Goos, M. (2010). Learning to teach mathematics with technology: A survey of professional development needs, experiences and impacts. Mathematics Education Research Journal, 22(1), 31-56. http://dx.doi.org/10.1007/BF03217558

Beogo, J. (2014). Accès à la formation continue des enseignants du primaire au Burkina Faso et la contribution des universités : les déterminants de la démarche individuelle (Thèse de doctorat, Université Paris-Est). Repéré à https://tel.archives-ouvertes.fr/tel-01130296/

Boatman, V. (2003). An investigation into the effects of long-term staff development on teacher perceptions and reading achievement of young children (Thèse de doctorat, University of North Texas). Repéré à https://digital.library.unt.edu/ark:/67531/metadc6149/m1/1/

Bowman-Perrott, L., Davis, H., Vannest, K., Williams, L., Greenwood, C. et Parker, R. (2013). Academic benefits of peer tutoring: A meta-analytic review of single-case research. School Psychology Review, 42(1), 39-55.

Brodeur, M., Gosselin, C., Legault, F., Deaudelin, C., Mercier, J. et Vanier, N. (2005). Prévention des difficultés d'apprentissage en lecture chez les enseignants de maternelle. Revue des sciences de l'éducation, 31(1), 33-54. https://doi.org/10.7202/012357ar 
Claesgens, J., Rubino-Hare, L., Bloom, N., Fredrickson, K., Henderson-Dahms, C., Menasco, J. et Sample, J. C. (2013). Professional development integrating technology: Does delivery format matter?. Science Educator, 22(1), 10-18.

Craig, H. J., Kraft, R. J. et Du Plessis, J. (1998). Teacher development. Making an impact. Repéré à http://documents.worldbank.org/curated/en/275761468758373557/pdf/multi-page.pdf

D’Amboise, G. et Audet, J. (1996). Le projet de recherche en administration. Un guide général à sa préparation. Québec, QC : Faculté des sciences de l'administration, Université Laval.

Dewi, R. S., Kultsum, U. et Armadi, A. (2017). Using communicative games in improving students' speaking skills. English Language Teaching, 10(1), 63-71. http://dx.doi.org/10.5539/elt.v10n1p63

Diallo, I. (1990). Problématique de la formation permanente des maîtres de l'enseignement de base (Mémoire de fin de stage inédit). Institut pédagogique du Burkina.

Edgerton, M. A. (2000). The effectiveness of a staff development program: Training teachers to use a code-based, explicit, and systematic reading instruction program (Thèse de doctorat inédite, University of North Carolina).

Ellis, A. K. (2001). Research on educational innovations (3e éd.). Larchmont, NY : Eye on Education.

Fontaine, S., Savoie-Zajc, L. et Cadieux, A. (2013). L'impact des CAP sur le développement de la compétence des enseignants en évaluation des apprentissages. Éducation et francophonie, 41(2), 10-34. http://dx.doi.org/10.7202/1021025ar

Glover, T. A., Nugent, G. C., Chumney, F. L., Ihlo, T., Shapiro, E. S., Guard, K., . . Bovaird, J. (2016). Investigating rural teachers' professional development, instructional knowledge, and classroom practice. Journal of Research in Rural Education, 31(3). Repéré à https://eric.ed.gov/?q=elementary+schools+teacher+development\&id=EJ1101917

Graves, M. F. (1986). Vocabulary learning and instruction. Review of Research in Education, 13, 49-89.

Gray, R. D., Drummond, A. J. et Greenhill, S. J. (2009). Language phylogenies reveal expansion pulses and pauses in Pacific settlement. Science, 323(5913), 479-483. http://dx.doi.org/10.1126/science.1166858

Guskey, T. R. (2000). Evaluating professional development. Thousand Oaks, CA : Corwin Press.

Guskey, T. R. (2001). The backward approach. Journal of Staff Development, 22(3), 60.

Guskey, T. R. et Huberman, M. (dir.) (1995). Professional development in education. New paradigms E̊ practices. New York, NY : Teacher College Press/Columbia University.

Harshman, J. et Yezierski, E. (2017). Assessment data-driven inquiry: A review of how to use assessment results to inform chemistry teaching. Science Educator, 25(2), 97-107.

Hattie, J. (2003, octobre). Teachers make a difference: What is the research evidence?. Communication présentée à la conference Building Teacher Quality : What Does the Research Tell Us?, Melbourne. Repéré à http://research.acer.edu.au/cgi/viewcontent.cgi?article=1003\&context=research conference 2003

Hill, K. K., Bicer, A. et Capraro, R. M. (2017). Effect of teachers' professional development from MathForward on students' math achievement. International Journal of Research in Education and Science, 3(1), 67-74. Repéré à http://files.eric.ed.gov/fulltext/EJ1126679.pdf

Houpert, D. (2005). En quoi la formation continue des enseignants contribue-t-elle au développement des compétences professionnelles?. Cabiers pédagogiques, (435). Repéré à http://www.cahiers-pedagogiques.com/En-quoi-laformation-continue-des-enseignants-contribue-t-elle-au-developpement-des-competences-professionnelles

Huberman, A. M et Miles, M. B. (1991). Analyse des données qualitatives. Recueil de nouvelles méthodes. Bruxelles : De Boeck.

Ilboudo, E. K, Compaoré, M., Ouedraogo, B., Somba, P., Kaboré, O., Ouedraogo, A., ... Kanoré, B. (2001). Revue de l'analyse sectorielle en éducation au Burkina Faso, 1994-1999. Repéré à http://unesdoc.unesco.org/images/0011/001177/117704Fb.pdf

Joyce, B. R. et Showers, B (1988). Student achievement through staff development. New York, NY : Longman. 
Kamano, P. J., Rakotomalala, R., Bernard, J.-M., Husson, G., et Reuge, N. (2010). Les défis du système éducatif Burkinabè en appui à la croissance économique (Document de travail $\mathrm{n}^{\circ}$ 196). Repéré à http://www-wds.worldbank.org/ external/default/WDSContentServer/WDSP/IB/2010/07/23/000020953 20100723160658/Rendered/PDF/ 558450FR0Les0defis0du0systeme.pdf

Karsenti, T. et Demers, S. (2000). Létude de cas. Dans T. Karsenti et L. Savoie-Zajc (dir.), Introduction à la recherche en éducation (p. 225-248). Sherbrooke, QC : Éditions du CRP.

Killion, J. (2002). What works in the elementary school. Results-based staff development. Repéré à http://www.friscoisd.org/docs/default-source/professional-development/whatworksinelem.pdf

Kulinna, P. H., McCaughtry, N., Martin, J. et Cothran, D. (2011). Effects of continuing professional development on urban elementary students' knowledge. Research Quarterly for Exercise and Sport, 82(3), 580-584. http://dx.doi.org/10.1080/02701367.2011.10599792

Kunsch, C. A., Jitendra, A. K. et Sood, S. (2007). The effects of peer-mediated instruction in mathematics for students with learning problems: A research synthesis. Learning Disabilities Research E Practice, 22(1), 1-12. http://dx.doi.org/10.1111/j.1540-5826.2007.00226.x

Kyelem, M. et Barro, M. (2007). Le système d'éducation de base burkinabé et ses entraves. Dans T. Karsenti, R.-P. Garry, J. Bechoux et S. Tchameni Ngamo, La formation des enseignants dans la francophonie : diversités, défis, stratégies d'action (p. 93-104). Repéré à http://www.rifeff.org/nouvelles download.php?f=1

L'Écuyer, R. (1990). Méthodologie de l'analyse développementale de contenu. Sillery, QC : Presses de l'Université du Québec.

Lane, L. M. (2003). The effects of staff development on student achievement (Thèse de doctorat inédite). University of Denver.

Le Breton, D. (2004). L'interactionnisme symbolique. Paris : Presses universitaires de France.

Lewis, E., Baker, D., Watts, N. B. et Lang, M. (2014). A professional learning community activity for science teachers: How to incorporate discourse-rich instructional strategies into science lessons. Science Educator, 23(1), 27-35. Repéré à http://files.eric.ed.gov/fulltext/EJ1034757.pdf

Lou, Y., Abrami, P. C. et D’Apollonia, S. (2001). Small group and individual learning with technology: A meta-analysis. Review of Educational Research, 71(3), 449-521. http://dx.doi.org/10.3102/00346543071003449

Lou, Y., Abrami, P. C., Spence, J. C., Poulsen, C., Chambers, B. et D’Apollonia, S. (1996). Within-class grouping: A metaanalysis. Review of Educational Research, 66(4), 423-458. http://dx.doi.org/10.3102/00346543066004423

Maïga, M. A. (1995). La formation en cours d'emploi : "principales causes de la faible fréquentation des séances par les maîtres de la province du Houet » (Mémoire de fin de stage inédit). École des cadres de l'animation et du contrôle pédagogique (ECAP).

Merriam, S. B. (1998). Qualitative research and case study applications in education (2 éd.). San Francisco, CA : Jossey-Bass.

National Early Literacy Panel. (2008). Developing early literacy. A scientific synthesis of early literacy development and implications for intervention. Repéré à http://www.lincs.ed.gov/publications/pdf/NELPReport09.pdf

National Institute of Child Health and Human Development (NICHHD). (2000). National reading panel. Teaching children to read: An evidence-based assessment of the scientific research literature on reading and its implications for reading instruction. Reports of the subgroups. Repéré à https://www.nichd.nih.gov/publications/pubs/nrp/Documents/report.pdf

OCDE. (1998). L'école à la page : formation continue et perfectionnement professionnel des enseignants. Paris : OCDE.

Richard, M., Bissonnette, S., Castonguay, M. et Gauthier, C. (2013). Enseignement explicite et réussite des élèves : la gestion des apprentissages. Saint-Laurent, QC : ERPI.

Rodriguez, A. J. (2010). Exposing the impact of opp(reg)ressive policies on teacher development and on student learning. Cultural Studies of Science Education, 5(4), 923-940. http://dx.doi.org/10.1007/s11422-010-9281-7

Rolheiser-Bennett, N. C. (1986). Four models of teaching: A meta-analysis of student outcomes (Thèse de doctorat inédite). University of Oregon. 
Rudnitsky, A., Etheredge, S., Freeman, S. J. M. et Gilbert, T. (1995). Learning to solve addition and subtraction word problems through a structure-plus-writing approach. Journal for Research in Mathematics Education, 26(5), 467-486. http://dx.doi.org/10.2307/749433

Solar, C. (dir.) (2001). Le groupe en formation des adultes. Bruxelles : De Boeck Université. http://dx.doi.org/10.3917/dbu.solar.2001.01

Sousa, A. D. et Donovan, J. F. (1993). Measuring the link by looking for other factors. Journal of Staff Development, 14(3).

Sparks, D. et Loucks-Horsley, S. (1989). Five models of staff development. Journal of Staff Development, 10(4).

Tessier, S. et D’Amboise, G. (2001). Facteurs de succès des PME en nouvelle économie. Repéré à http://www.fsa.ulaval.ca/sirul/2001-013.pdf

UNESCO. (2003). Pour un renforcement des capacités des institutions de formation des enseignants en Afrique subsabarienne. Essai d'analyse de la situation et proposition pour l'action. Paris : UNESCO.

UNESCO. (2004). Éducation pour tous. L'exigence de qualité. Rapport mondial de suivi sur l'EPT 2005. Repéré à http://unesdoc.unesco.org/images/0013/001374/137403f.pdf

UNESCO. (2005). Comprendre ce qu'est la qualité. Dans UNESCO, Rapport de suivi sur l'éducation pour tous (p. 30-41). Repéré à http://www.unesco.org/education/gmr_download/chapter1_fr.pdf

UNESCO. (2014). Rapport mondial de suivi sur l'EPT 2013-2014. Enseigner et apprendre : atteindre la qualité pour tous. Repéré à http://unesdoc.unesco.org/images/0022/002261/226157f.pdf

Williams-McMillan, Y. et Hauser, G. M. (2014). The impact of a system-wide community college professional development program on pedagogical practice: An assessment of faculty perspectives. International Journal of Arts E Sciences, 7(2), 617-627. Repéré à http://www.universitypublications.net/ijas/0702/pdf/P4RS32.pdf

Womack, J. T. S. (2004). Journey to change: the effects of job-embedded, on-going staff development on teacher performance and student achievement in the area of writing in Russels County schools (Thèse de doctorat inédite). Auburn University.

Zambak, V. S., Alston, D. M., Marshall, J. C. et Tyminski, A. M. (2017). Convincing science teachers for inquiry-based instruction: Guskey's staff development model revisited. Science Educator, 25(2), 108-116.

\section{Pour citer cet article}

Djibo, F. (2017). L'efficacité de la formation continue des enseignants du primaire : le cas du Burkina Faso. Formation et profession, 25(2), 35-48. http://dx.doi.org/10318162/fp.2017.330 